\title{
Ouamuamua (A/2017U1), Panspermia, and Intelligent Life in the Universe
}

\author{
N.C. Wickramasinghe ${ }^{1,2,3}$, D.T. Wickramasinghe ${ }^{3,4} \&$ E.J. Steele ${ }^{2,5}$ \\ ${ }^{1}$ Buckingham Centre for Astrobiology, University of Buckingham, Buckingham MK18 1EG, UK \\ ${ }^{2}$ Centre for Astrobiology, University of Ruhuna, Matara, Sri Lanka \\ ${ }^{3}$ Institute for the Study of Panspermia and Astrobiology, Gifu, Japan \\ ${ }^{4}$ College of Physical and Mathematical Sciences, Australian National University, Canberra, Australia \\ ${ }^{5}$ CY O'Connor ERADE Village Foundation, Piara Waters, WA, Australia
}

\begin{abstract}
We examine a range of arguments relating to the existence of extraterrestrial life and the possible distribution of intelligent life in the galaxy. It has recently been reported that the object (Ouamuamua (A/2017U1)) that transited the solar system in a hyperbolic orbit exhibits certain features that cannot be readily explained on the basis of it being a naturally-occurring comet or asteroid. The interesting conjecture by Bialy and Loeb that Ouamuamua (A/2017U1) could be an artificial construct of an alien civilization is re-examined. The possibility that Ouamuamua may be a dark cometary body, in which unobserved gaseous emissions may have led to departures from a Keplerian orbit, remains a more conservative alternative possibility. We discuss the implications of the discovery of light sails or similar artifacts, as proposed by Bialy and Loeb, in relation to the validity of galaxy-wide panspermia and the prevalence of life throughout the universe.
\end{abstract}

Keywords: Exobiology, panspermia, comets, extraterrestrial intelligence, SETI

\section{Frequency of Intelligent Life}

Perhaps the most profound of all scientific and philosophical questions ever been asked relate to our place as sentient creatures in the cosmos. Could we humans be possibly alone as intelligent living creatures in the Universe poised atop a pyramid of life, which itself is hypothesized to have arisen by random processes on Earth? In regard to the transition from non-living organic molecules to the simplest self-replicating bacterium, we have argued elsewhere that a priori probabilities are so vanishingly small that timescales and distance-scales of cosmological magnitude are minimally required (Hoyle and Wickramasinghe, 1982). We have also discussed at length the details of astronomical and biological evidence that support this conclusion (Steele, et al, 2018). In relation to the possible emergence of human intelligence or super-human intelligence outside the Earth, we could be encouraged by recent discoveries of habitable exoplanets which probably exist in their tens or even 100's of billions in the galaxy. It may therefore be reasonable to consider the process of the spread of life by panspermia amongst these planets, with an evolution converging to higher levels of intelligence. In such a case, we might be encouraged to look for evidence of extraterrestrial intelligence as for instance in SETI-type projects.

In the famous Drake equation the number $N$ of advanced civilizations within our reach is given as the product of several factors:

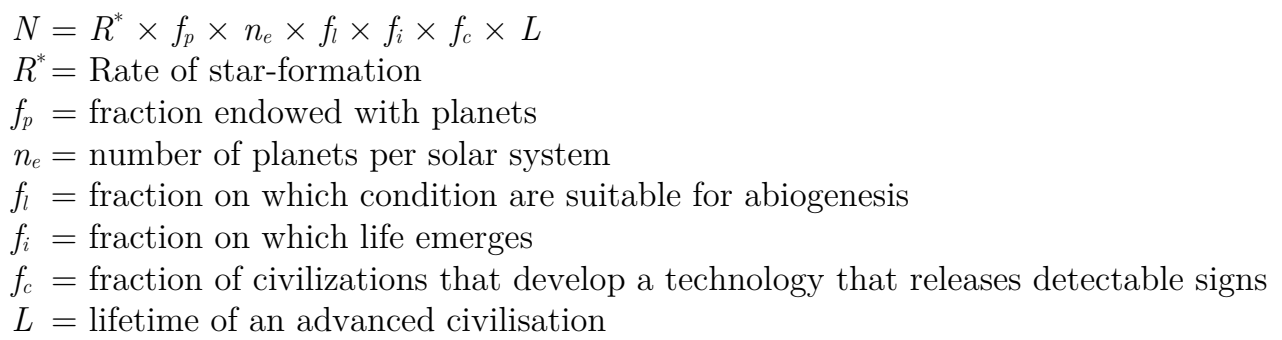

Of all the factors in this expression the most important is $f_{l}$ the probability of life emerging by spontaneous generation of localized abiogenesis. If as we argue $f_{l}=0$ (Steele et al, 2018) then it follows 
that $N=0$ and SETI and similar projects must be finally laid to rest. Only if life is a cosmic phenomenon (Hoyle and Wickramasinghe, 1982) can the situation be rescued. But with the one proven instance of life on Earth, and in view of our earlier comments on the implausibility of Earth-bound abiogenesis, we should consider replacing $f_{l}$ by $f_{l p}$ where

$f_{l p}=$ initiation of cosmic life by panspermia

and the deferring the ultimate origin of life to a distant cosmological past.

The factor $f_{l p}$ is difficult to estimate a priori, but the recent studies of Ginsburg et al (2018) suggest that as many as $10^{13}$ (10 trillion) planetoids or free-floating life-bearing bodies with dimensions in excess of $1 \mathrm{~km}$ bearing life could have been exchanged between galaxies similar to the Milky Way over the age of the Universe. This would make a tentative value of $f_{l p} \sim 1$ to be a reasonable guess.

In the so-called standard theories of abiogenesis the emergence of life in situ on any individual habitable planet involved an unknown probability factor. Understanding the detailed steps leading to abiogenesis, including the precise arrangements of biochemical monomers necessary for the operation of a living cell, have continued to elude scientists for well over half a century (Deamer, 2016). Our own preference for a cosmic/cosmological origin of life is consistent with a precisely zero value of probability for any independent planetary origin of life. We accept the premise that the steps that led to life's first emergence are tucked away in the mists of our cosmological past.

One particular cosmological schema that has been suggested by Gibson et al (2011) involves chemical processes that occurred in a vast multitude of primordial planets 2-20 million years after the Big Bang (See also Wickramasinghe et al. (2010, 2012) and Joseph \& Schild (2010)).

Recent cosmological studies, however, would place this event somewhat later at $200 \mathrm{Myr}$, after the Big Bang after the first stars would have formed, thus producing metals that could have enabled the formation of rocky planets with water-rich/organic domains that could have presumably led to the first origin of life (Shchekinov et al. 2013).

We do not regard this particular schema as uniquely representing the way things really happened but it arguably remains a possibility. The main point to make here is that life is unquestionably, in our view, a cosmological phenomenon, one that is utterly impossible to reproduce on any smaller astronomical or planetary scale.

\section{Directed Panspermia and Cosmic Biology vs Abiogenesis}

For the origin of life on the Earth the idea of directed panspermia was first suggested by Francis Crick and Orguel (1974), and a few years afterwards more realistic theories of cosmic biology and cometary panspermia were introduced and developed by Hoyle and Wickramasinghe $(1978,1981,2000)$. The early trend to discount such ideas were rampant when there was no convincing evidence for either the existence of habitable exoplanets or the operation of the process of horizontal gene transfer between organisms (see Steele et al, 2018). It was also possible in the 1980's to entertain a vain hope of abiogenesis as being a process that may have occurred during the first 0.5 billion years of geological history, up to the time when the first definite microfossils were found in terrestrial rocks. This situation has changed in recent years with the earliest evidence of microbial life being discovered in rocks dating back to 4.2-4.3 billion years ago, life thus appearing on the Earth at the very first moment it could survive (see references in Steele et al, 2018).

We have already referred to the profusion of exoplanets in "Goldilocks Zones" in the galaxy that might serve as congenial homes for life. Further we now know that viruses can act as agents for the transfer of genes across species, and that they play a crucial role in evolution. In the many gene sequencing programmes that have been carried out since 2001, viruses (including retroviruses, and segments of viruses) have shown up in great abundance. Panspermia, directed or otherwise, has become an idea that can no longer be dismissed as idle speculation. The requirement for biological evolution to be necessarily contained within the terrestrial biosphere has effectively disappeared.

Perhaps most importantly, all abiogenesis experiments that have been carried out thus far have dismally failed. It is difficult now to claim that arriving at the specific super-astronomical level of complexity of the simplest living bacterium is a chemical imperative. On the contrary, if we start from non-living organic molecules (monomers), producing even the simplest form of primitive cellular life involves overcoming an improbability hurdle of super-astronomical magnitude. Circumventing this 
problem would require access to a "probability space" extending over cosmological dimensions of space and time, and this can certainly not happen on any confined planetary scale.

The alternative theory of a cosmic or cosmological origin of life, along with its fullest compliment of evolutionary possibilities dispersed in the form of information carried in viruses, viroids and bacteria, now has a formidable body of evidence in its favour (Steele et al, 2018). A corollary would be that the evolution of life everywhere in the universe marches inexorably towards higher and higher levels of sophistication and expressed intelligence.

\section{Dark Comet Hypothesis}

In a recent paper it was pointed out that if each one of the 100 billion or so exoplanetary systems that are currently estimated to exist in the Galaxy are losing comets a rate of $\sim 10$ per year (an estimate considered appropriate for the solar system) the total number of interstellar comets in our vicinity is $\sim 3$ $\times 10^{21}$ (Wickramasinghe, et al, 2018a). The fact that only one hyperbolic comet, viz Ouamuamua (A/2017U1), has thus far been observed therefore demands an explanation. One clue may be found in the apparently dark surface (low albedo) exhibited by Ouamuamua.

The exceedingly low values of albedo appropriate for the porous coal-like surfaces of evolved comets may explain the appearance of Ouamuamua whilst also accounting for the perceived rarity of hyperbolic comets (Wickramasinghe et al, 2018b). A similar explanation may also be relevant for accounting for an observed deficit of comets in Halley-type orbits (Wallis \& Wickramasinghe, 1985; Napier et al, 2004). Such a population of dark-crusted organic comets has been regarded as posing an unseen collision threat with the Earth, a threat extending over geological timescales. Similarly, there may have been many dark hyperbolic comets that have traversed the inner solar system in recent times without ever attracting our attention. The dark interstellar comet hypothesis still remains be a viable "conventional" explanation for Ouamuamua, although it would certainly be prudent to keep other more exotic options open for discussion.

The dark comet model we prefer would consist of an organic-crusted exterior of low albedo (like Comet $\mathrm{P} /$ Halley and other comets) with an icy interior. Thus, upon reaching perihelion, it would outgas mostly $\mathrm{H}_{2} \mathrm{O}$, so maintaining consistency with observations, without necessarily requiring a thin light-sail as suggested by Bialy \& Loeb (2018).

Besides a population of transiting hyperbolic comets (including dark comets) it is also possible for extrasolar bodies to occasionally undergo capture via three-body gravitational interactions and thence become trapped in our Solar system. If so, this provides an empirical testing ground for the ideas discussed in this paper. It will also help ameliorate the criticism of those who might like to maintain that cosmic panspermia is not testable. By locating such extra-solar objects in the very "backyard" of the Solar system, we can potentially investigate the theory of cosmic panspermia in a rigorous manner. Lingam and Loeb (2018) have estimated the number of such captured objects of a size comparable to Oumuamua to be $\sim 6000$, using analytical and numerical models. This would give a fair chance of their future discovery through space exploration.

\section{The Artificial Object Hypothesis for Ouamuamua}

At the present time, it appears more or less certain that the totality of observations of Ouamuamua cannot easily be reconciled with a cometary hypothesis. Many questions therefore remain as to the nature and origin of this object. A recent study by Micheli et al (2018) has shown that Ouamuamua's orbit exhibits significant departures from a Keplerian orbit, thus suggesting an effect such as might arise from the action of radiation pressure from the sun. Similar departures are well known for comets with dust tails, but for Ouamuamua with no evidence of significant outgassing or a dust tail this phenomenon could be seen as posing a problem.

Bialy and Loeb (2018) have recently made the intriguing suggestion that the same effect as that of a dust tail could be achieved if Ouamuamua was a thin rectangular "light sail" with a mass to area ratio of $0.1 \mathrm{~g} \cdot \mathrm{cm}^{-2}$ on which radiation pressure could effectively act so as to overcome the effect of gravity. Whilst this is theoretically possible it is equally possible to think in terms of an ellipsoidal shell of high elongation (eccentricity) made from ultrathin material that might also fit the bill. One might for 
example envision a such a shell made of ultrathin graphitic or $\mathrm{C}_{60}$ or carbon fibre-like material, where a modest increase of pressure of entrapped gas over the external pressure may be thought able to maintain an ellipsoidal shape.

\section{Dynamics of an Ultrathin Ellipsoid in the Solar Vicinity}

It is possible to model the general features of the dynamics of single layered mesh of carbonaceous dust grains (either a flat sheet or ellipsoidal "balloon") based on the behaviour of individual dust grains in the solar environment with a view to explaining the orbital data of Micheli et al (2018).

Consider a spherical carbon (graphite, graphene) sphere of radius $r$, density s located at a distance $R$ from a star similar to the Sun. At distances $R$ from the star where $R>>R_{\text {Sun }}$ the outward radiation pressure force experienced by the grain is given by

$$
P=\frac{L}{c} \pi r^{2} Q \frac{1}{4 \pi R^{2}}
$$

where $L$ is the bolometric luminosity of the star $c$ is the velocity of light, $r$ is the radius of the grain and $Q$ is the mean efficiency for radiation pressure (averaged over the stellar spectrum). The oppositely directed gravitational attractive force is

$$
F=G \frac{m M}{R^{2}}=G \frac{4}{3} \frac{\pi r^{3} s M}{R^{2}}
$$

where $G$ is the universal gravitational constant, $M$ is the mass of the star and $s$ is the specific gravity of the grain material. Thus we get a radiation pressure to gravitational force ratio:

$$
\frac{P}{F}=\frac{3 Q}{16 \pi r s c G} \frac{L}{M}=5.73 Q /\left(r / 10^{-5} \mathrm{~cm}\right)
$$

using $L / M \approx 3.83 / 1.99$, appropriate to the Sun, $Q$ values appropriate for graphite spheres of radii $r=$ $0.1 \mu \mathrm{m}$ (eg Wickramasinghe, 1973,) and material specific gravity $s \approx 1$.

Based on the measured optical constants of graphite it can be shown (from Mie calculations for particles of diameter 0.1-0.3micron to estimate $Q$ ) that the ratio of radiation pressure force to gravity in the vicinity of the sun could be in excess of 5 (Wickramasinghe, 1973). If the parent star of our putative alien civilization was a luminous red giant, this ratio could be further increased by a significant factor.

Our preferred variant of the Bialy-Loeb conjecture would be to replace their thin sheet model by an ultra-thin ellipsoidal spacecraft that can be driven by the pressure of sunlight; and such a spacecraft could even bear the burden of a few times its mass in the form of light cargo.

This immediately raises the spectre of an advanced alien civilization that is capable of constructing and launching such a spaceship. It is interesting to note in passing that the image conjured up for Ouamuamua is strikingly reminiscent of Rama in Arthur C. Clarke's science fiction novel "Rendezvous with Rama" (Clarke, 1973). In this story a thirty-mile-long cylindrical spaceship is detected travelling on a course to traverse our solar system.

\subsection{Radiation Pressure Expulsion from a Parent Star}

For a structure satisfying the condition $P / F>1$ the radial acceleration outward for the parent star is given by

where $\alpha=P / F-1$. Integration of this leads to

$$
\ddot{R}=\alpha \frac{G M}{R^{2}}
$$

$$
\dot{R}^{2}=2 \alpha G M\left[\frac{1}{R_{0}}-\frac{1}{R}\right]
$$

$R_{0}$ being the radial distance from which the particle was expelled. As $R \rightarrow \propto$ the asymptotic velocity at which the particle leaves the star is

$$
V=\sqrt{\frac{2 \alpha G M}{R_{0}}}
$$

Taking $\alpha=0.5, R_{0}=1 A U$ we get $V=3 \times 10^{6} \mathrm{~cm} / \mathrm{s}$ implying a travel time of $100 \mathrm{pc}$ in 3 million years. As Bailey and Loeb (2018) correctly state an object moving at this speed is not much slowed by collisions with the extremely tenuous interstellar gas. It is also interesting to note that a speed of 
$30 \mathrm{~km} / \mathrm{s}$ is close to the speed that can be achieved by chemical propulsion, and also to the hyperbolic excess velocity ( $v$ at infinity) observed for Oumuamua $(26 \mathrm{~km} / \mathrm{s})$. This would seem to illustrate two points:

(i) Our results are consistent with observations,

(ii) More daringly we might assert that civilizations can use radiation pressure driven spacecraft instead of rockets, since the former could be lighter and would not need to carry their own fuel.

\section{From Where Did Ouamuamua Come?}

If Bialy and Loeb's (2018) speculation is to be taken seriously we must infer that the architect of Ouamuamua (or what it originally was) hails from a technologically advanced civilization located on an exoplanet perhaps tens or even hundreds of parsecs away. How plausible would such a proposition be? And does this idea not represent a radical departure from the canon of "respectable" science?

The nearest candidate exoplanet from which Ouamuamua may have originated could be conjectured to be Proxima b, the planet recently discovered to orbit the star Proxima Centauri at a distance of 1.3 parsecs away (Angelade-Escude, 2016). Another interesting conjecture is that there exists a habitable planet around the "solar twin" HD186306, a star that is believed to have formed in the same stellar nursery as the sun 5 billion years ago (Adibekyan et al, 2018). This star is currently located at a distance of $\sim 400 \mathrm{pc}$; but it may well have been very much closer in the early history of the solar system, in particular during the epoch of Late Heavy Bombardment of Earth. It has recently been discovered that that earliest microbial life on the Earth dates back to this epoch some 4.1 billion years ago (Bell et al, 2015). Thus if the star HD186306 was sufficiently close-by at this time and if it was endowed with a habitable planet one might speculate that microbiota may well have been exchanged between this planet and Earth by means of collisions. A similar process of collisional transfer between planets was discussed earlier by Wallis and Wickramasinghe (1995, 2004). Such a process would have ensured that the two now widely separated planets possess a biochemical unity.

\section{$7 \quad$ Distribution of Advanced Life}

If one is constrained to think in terms of an Earth-centred process of abiogenesis the recurrence of the same event elsewhere will essentially constitute a double miracle. In this case the further biological developments leading to technologically advanced species capable of launching Ouamuamua-type vehicles will be virtually impossible. A cosmic origin of all life, accompanied by convergent patterns of genetic evolution, would lead naturally to an abundance of sites where intelligence and technology would have arisen and developed. If, say, $0.1 \%$ of all the habitable planets in the Galaxy had developed in a parallel way to Earth, the number of such planets will be $\sim 10^{8}$. Distributed over the volume of the interstellar medium $\sim 3 \times 10^{10} \mathrm{pc}^{3}$, this gives a mean separation of technologies of $\sim 300 \mathrm{pc}$.

On the Earth the emergence of higher life and intelligence that is capable of embarking on space exploration has been relatively recent, perhaps going back no further than say 4000 years. This is still only a minute fraction $\sim 10^{-6}$ of the time that life has existed on Earth, and thus could be interpreted as a factor that severely limits the number of intelligent galactic civilizations within our immediate reach. A further limiting factor could be the natural lifetime of an advanced civilization whenever and wherever they arose. If our human trends and tendencies prevail universally it is likely that there is a natural expiration date of advanced technologies determined by an inescapable folly written into our cosmic genes. The risk of annihilation through nuclear or biological warfare has also to be augmented by the risk of destruction by comet or asteroid impacts.

In conclusion, we note that the emergence of intelligence and super-intelligence on alien exoplanets and its persistence for long enough to enable the launching of interstellar spacecraft of the type proposed by Bialy and Loeb (7) must regarded as a virtual certainty. This would be a natural consequence of the theory that life itself is a cosmic phenomenon, with all its evolution and evolutionary potential already fully determined and established over cosmological timescales and cosmological dimensions. If, on the other hand, one is constrained to think in terms of abiogenesis on Earth as a localised phenomenon that occurred against the most impossible odds the chance of this event being repeated elsewhere would effectively constitute a miracle. 


\section{Cosmological Constraints}

Recently Lin and Loeb (2015) have attempted to calculate the degree of clustering in domains of inhabited planets in the Universe according to both in situ abiogenesis and panspermia. Their arguments essentially assume a non-zero value for $f_{l}$, the probability of life emerging by planetary abiogenesis, along with various values of $f_{l p}$ (in our terminology). If $f_{l}$ is small but finite the precise value of $f_{l p}$ determines a scale of clustering that could be looked for when observations do become available for the distribution of life in the universe. If $f_{l}=0$ however, that is to say life is a cosmic phenomenon, shaped by panspermia and the mixing of genes over a cosmological scale, the analysis of Lin and Loeb does not directly apply.

In another interesting speculation Ginsburg, Lingam and Butler (2019) compares the large-scale structure and the implied information content of the entire universe to the neural connections of an animal brain. The correspondences are indeed striking, suggesting that the one is seemingly a close mapping of the other. However, despite its enormous size the cosmos is evidently many orders of magnitude less efficient at computation than the brains resident on Earth. This illustrates yet again the super-astronomical information content and capacity of life.

Finally, if we adopt the currently accepted concordance Big Bang model of the universe, our observable horizon is at a radius of 46 billion light years (see Tamara and Lineweaver (2004)). The true extent of the universe of course depends on the precise spatial curvature of the universe and constraints from MWB radiation observations point to the universe being flat to within $0.4 \%$. The universe may therefore be at least a factor of a few hundred larger, and may even be infinite in extent if it turns out that the universe is precisely flat. If life originates as a highly improbable event as is implied by our present arguments, it is likely that these events will be localized within disjoint "life-domains" in the Universe. The cosmological domain in which we happen to find ourselves defines the nature of our own idiosyncratic cosmic biology, including its basic biochemistry, genetic code as well as its full range of genetic diversity - a range that is only incompletely expressed on the Earth.

Acknowledgement. We are grateful to an anonymous reviewer for valuable comments on an earlier version of this paper.

\section{References}

1. Adibekyan, V. et al., 2018, Astron\&Astrophys, 619, Nov 2018 (DOI:10.1051/0004-6361/201834285)

2. Anglada-Escude, G. Et al, 2016, Nature, 536, 437

3. Bell, E.A., et al, 2015, Proc.Nat.Acad.Sci.USA, 112, 14518-14521

4. Bialy, S. and Loeb, A., 2018, ApJ Lett., 868(1), L1

5. Clarke, A.C., 1973. Rendesvouz with Rama, Bantam Books

6. Crick, F. H. C., and Orgel, L. 1973, Icarus, 19, 341

7. Deamer, 2016, First life, Univ.Calif.Press

8. Gibson, C., Schild, R.E., Wickramasinghe, N.C., 2011. Int J. Astrobiol., 10(2), 81

9. Ginsburg, I., Lingam, M., and Loeb, A. 2018, Astrophys. J. Lett., 868:L12

10. Ginsburg, I., Lingam, M., and Butler, M. 2019, Advances in Astrophysics, 4(3), 83; https://dx.doi.org/10.22606/adap.2019.43001 83

11. Hoyle, F. and Wickramasinghe, N. C. 1978, Lifecloud: The origin of life in the galaxy, Dent, London

12. Hoyle, F. and Wickramasinghe, N. C. 1981, in Comets and the Origin of Life, ed. C. Ponnamperuma, Reidel, Dordrecht

13. Hoyle, F. and Wickramasinghe, N. C. 1982, Evolution from Space, Dent, London

14. Hoyle, F. and Wickramasinghe, N.C., 2000, Astronomical Origins of Life (Kluwer Academic Publ)

15. Joseph, R. and Schild, R., 2010, J. Cosmol., 5:1040-1090

16. Lin, H.W. and Loeb, A., 2015, Ap.J.Lett, 810, L3

17. Lingam, M. and Loeb, A., 2018, Astron. J., 156(5):193

18. Loeb, A., 2014, Int. J. Astrobiol., 13(4):337-339

19. Micheli, M. et al, 2018, Nature, 559, 223

20. Napier, W.M., Wickramasinghe, J.T., Wickramasinghe, N.C., 2004, Mon.Not.R.A.S., 355, 191 
21. Shchekinov, Y. A., Safonova, M., and Murthy, J., 2013, Astrophys. Space Sci., 346(1):31-40

22. Steele, E.J. et al, 2018, Progress in Biophysics and Molecular Biology 136 (2018) 3e23

23. Wallis, M.K. and Wickramasinghe, N.C., 1985, Mon.Not.R.A.S., 216, 453

24. Wallis, M.K. and Wickramasinghe, N.C., 1995, Earth Planet.Sci.Lett., 130, 69-73

25. Wallis, M.K. and Wickramasinghe, N.C., 2004, Mon.Not.R.A.S., 348, 52-61

26. Wickramasinghe, N.C. et al, 2018a, Advances in Astrophysics, 3 (1)

27. Wickramasinghe, N.C. et al, 2018b, Advances in Astrophysics, 3 (2)

28. Wickramasinghe, N.C., 1973, Light Scattering by Small Particles with Applications in Astronomy (Wiley, NY)

29. Wickramasinghe, N.C., Wickramasinghe, D.T., Steele, E.J., 2018, Astrophys.Sp.Sci., 363, 244

30. Wickramasinghe, N. C., Wallis, J., Wallis, D. H., Schild, R. E., Gibson, C.H., 2010, Evolution of primordial planets in relation to the cosmological origin of life. Proc. SPIE, 7819, 78190D

31. Wickramasinghe, N. C., Wallis, J., Wallis, D. H., Schild, R. E., Gibson, C.H., 2012, Astrophys. Space Sci., $341(2), 295$ 